This is a postprint version of the following published document:

Morales Céspedes, M.; Sánchez-Fernández, M.; García Armada, A.;(2015). Experimental Evaluation of Blind Interference Alignment. Vehicular Technology Conference (VTCSpring), 2015 IEEE , pp.1-5. Available in http://dx.doi.org/10.1109/VTCSpring.2015.7145832

(C2015. IEEE. Personal use of this material is permitted. Permission from IEEE must be obtained for all other uses, in any current or future media, including reprinting/republishing this material for advertising or promotional purposes, creating new collective works, for resale or redistribution to servers or lists, or reuse of any copyrighted component of this work in other works. 


\title{
Experimental Evaluation of Blind Interference Alignment
}

\author{
Máximo Morales Céspedes, Matilde Sánchez Fernández, Ana García Armada \\ Universidad Carlos III de Madrid - Spain \\ Department of Signal Theory and Communications \\ e-mail: maximo@tsc.uc3m.es, mati@tsc.uc3m.es, agarcia@tsc.uc3m.es
}

\begin{abstract}
An experimental evaluation of Blind Interference Alignment (BIA) over a hardware platform is presented in this work. In contrast to other transmission techniques such as Linear Zero Forcing Beamforming (LZFB) or Interference Alignment (IA), BIA achieves a growth in Degrees of Freedom (DoF) without channel state information at the transmitter (CSIT). A real implementation based on Orthogonal Frequency Division Multiplexing (OFDM) and LTE parameters is implement on a testbed made up of a transmitter equipped with two antennas and two users equipped with a reconfigurable antenna each. Furthermore, a full CSIT technique such as LZFB is also implemented for comparison purposes. First, the theoretic achievable rates are obtained for both techniques. After that, the bit error rate of both schemes is evaluated regarding the achieved sum-thorughput.
\end{abstract}

\section{INTRODUCTION}

The increasing demand of high data rates resulted in a growing interest in Multiple-Input Multiple-Output (MIMO) systems. Several techniques such as Linear Zero Forcing Beamforming (LZFB) or Interference Alignment (IA) have been proposed as a means to achieve a significant increment of Degrees of Freedom (DoF), also known as multiplexing gain, regarding orthogonal schemes. Unfortunately, these schemes are usually based on the knowledge of the Channel State Information at the Transmitter (CSIT). This requirement involves high-capacity backhaul links and instantaneous feedback, which consume a large amount of network resources. Consequently, the implementation of schemes based on CSIT results challenging in a real implementation.

Recently, a novel technique called Blind Interference Alignment (BIA) that achieves a growth in DoF without the need for CSIT was proposed in [1]. BIA is usually based on reconfigurable antennas that switch their radiation pattern among a fixed number of preset modes [2]. As demonstrated in [1], BIA achieves $\frac{N_{t} K}{N_{t}+K-1}$ DoF in the Multiple Input Single Output Broadcast Channel (MISO-BC) where the transmitter is equipped with $N_{t}$ antennas and there are $K$ active users. Besides, BIA not only achieves more DoF than orthogonal techniques such as TDMA, it is demonstrated in [3] that it achieves the outer bound of this setting in absence of CSIT. On the other hand, since the DoF metric assumes $S N R \rightarrow \infty$, it is not clear if BIA overcomes the performance of other more simple techniques at low SNR regime. In contrast, the absence

This work has been partially funded by research projects COMONSENS (CSD2008-00010), and GRE3N (TEC2011-29006-C03-02). of CSIT, and therefore of feedback, the use of BIA allows to relax the synchronization requirements regarding LZFB or IA.

To understand the impact of these techniques on practical wireless networks, it is necessary to evaluate their performance in real scenarios beyond simple channel models often used in simulations-based approaches (e.g. spatially uncorrelated channels, perfect synchronization, channel coding...). Unfortunately, due to the costs associated to the required hardware setup, the literature about real implementations of novel schemes is scarce. An IA offline evaluation of MIMO-OFDM 3-user interference channel is presented in [4]. It is demonstrated that the theoretical rates are achievable in practice. However, due to the offline evaluation, practical issues such as synchronization or hardware impairments are not taken into account. A study of IA performance focused on 3-user interference channel is presented in [5]. This work identifies the main practical issues that degrade the IA performance in a real implementation, concluding that imperfect CSIT is the key limiting factor. The performance of LZFB and Block Diagonalization (BD) is evaluated in [6]. It is shown that the theoretical rates are only achievable when strong channel coding is employed. Therefore, since the transmitted symbols have to be heavily coded, the achieved multiplexing gain is futile. The performance of BIA is compared with transmission based on time division in the implementation presented in [7]

In this work BIA experiments are carried out in the $5 \mathrm{GHz}$ band on the MIMO-OFDM testbed of [8] based on a LTE configuration. A MISO BC where the transmitter is equipped with two antennas that serve two users equipped with a set of two antennas connected to only one RF chain, which composes a reconfigurable antenna, each is taken into consideration. Therefore, each user can switch among two different radiation patterns. By applying BIA over this configuration, each user attains $\frac{2}{3}$ DoF without the need for CSIT. With the aim to compare the performance of BIA with a full CSIT technique, LZFB is also implemented taking into account the costs associated to CSIT knowledge. The proposed experiments are based on a typical configuration of a LTE system for a bandwidth of $5 \mathrm{MHz}$, where a turbocode with coding rate of $\frac{2}{3}$ is employed for channel coding.

The remainder of this paper is structured as follows. In Section II the system model is stated for BIA and LZFB schemes. Section III is devoted to describe the measurement set-up and Section IV develops the frame structure of each 
scheme. The theoretical and measured results are shown in Section V. Finally, concluding remarks are given in Section IV.

\section{SySTEM MOdEL}

The system model assumes a MISO BC where the transmitter is equipped with $N_{t}$ antennas that serve $K$ active users. Each user can switch among $N_{t}$ preset modes of its reconfigurable antenna. An OFDM approach with $N_{\text {ofdm }}$ subcarriers is employed in this work. Therefore, the signal received by the $k$-th user on the $p$-th subcarrier at time $t$ can be written as

$$
y_{p}^{[k]}(t)=\mathbf{h}_{p}^{[k]}\left(m^{[k]}(t)\right)^{T} \mathbf{x}_{p}(t)+z_{p}^{[k]}(t),
$$

where $\mathbf{h}_{p}^{[k]}\left(m^{[k]}(t)\right) \in \mathbb{C}^{N_{t} \times 1}$ is the channel vector on the $p$ th subcarrier between transmitter and user $k$ corresponding to the $m$-th radiation pattern of this user, $\mathbf{x}_{p}(t) \in \mathbb{C}^{N_{t} \times 1}$ are the transmitted symbols on the $p$-th subcarrier, and $z^{[k]}(t)$ is the thermal noise on the $p$-th subcarrier of each antenna, which is assumed complex Gaussian with zero mean and unit variance. The channel input is subject to an average power constraint $E\left\{\left\|\mathbf{x}^{[n]}[t]\right\|^{2}\right\} \leq P$.

\section{A. Blind Interference Alignment}

The key idea of BIA is to align the interference by exploiting the channel correlations generated by a predefined pattern of channel preset modes, which will be denoted as supersymbol from now on. Following the lines of [1], the supersymbol structure for the $K$ users and $N_{t}=2$ antennas configuration is shown in Figure 1. It is composed of two parts; Block 1 where the transmitter sends the desired symbol simultaneously to every user, and Block 2 where the symbols are transmitted in an orthogonal fashion.

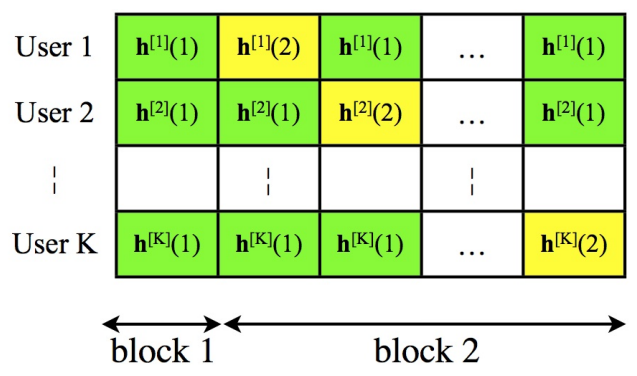

Fig. 1. Supersymbol structure of BIA for $N_{t}=2$ and $K$ users.

The beamforming vectors are determined by the supersymbol structure. During the first symbol extension, the transmitter sends $N_{t}$ different symbols simultaneously to each of the $K$ users. During the subsequent time slots the desired $N_{t}$ symbols of each user are transmitted independently. Since the transmitter is equipped with 2 antennas in the proposed testbed, in order to generate an equation system to decode $N_{t}=2$ symbols, each user has to receive its corresponding symbols during the two preset modes of the reconfigurable antenna. In other words, each user exploits a full rank matrix
$\mathbf{H}_{p}^{[k]}=\left[\mathbf{h}_{p}^{[k]}(1), \mathbf{h}_{p}^{[k]}(2)\right]^{T}$ with the aim to decode its desired symbol. On the other hand, taking advantage of the orthogonal transmission employed during Block 2, the interference symbols transmitted to the rest of user can be measured by the user of interest and subtracted afterwards. Thus, for the $N_{t}=2$ transmit antennas case, the beamforming matrix is given by

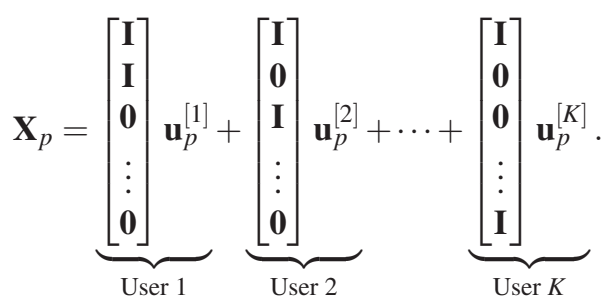

where $\mathbf{u}_{p}^{[k]}=\left[u_{1, p}^{[k]}, u_{2, p}^{[k]}\right]^{T}$ is the vector that contains the $N_{t}=2$ desired symbols of the $k$-th user on the $p$-th subcarrier.

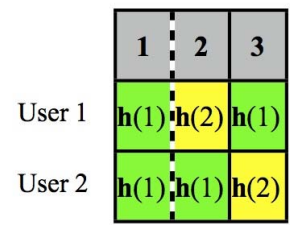

$$
\mathbf{X}_{p}=\left[\begin{array}{l}
\mathbf{I} \\
\mathbf{I} \\
\mathbf{0}
\end{array}\right] \mathbf{u}_{p}^{[1]}+\left[\begin{array}{l}
\mathbf{I} \\
\mathbf{0} \\
\mathbf{I}
\end{array}\right] \mathbf{u}_{p}^{[2]}
$$

Fig. 2. Supersymbol structure for $N_{t}=2, K=2$.

For illustrative purposes, we show the specific case considered in this work. The supersymbol and transmission strategy are shown in Figure 2. In this case, the signal received by user 1 is given by

$$
\left[\begin{array}{c}
y_{p}^{[1]}(1) \\
y_{p}^{[1]}(2) \\
y_{p}^{[1]}(3)
\end{array}\right]=\underbrace{\left[\begin{array}{c}
\mathbf{h}_{p}^{[1]}(1)^{T} \\
\mathbf{h}_{p}^{[1]}(2)^{T} \\
\mathbf{0}
\end{array}\right]}_{\text {rank=2 }} \mathbf{u}_{p}^{[1]}+\underbrace{\left[\begin{array}{c}
\mathbf{h}_{p}^{[1]}(1)^{T} \\
\mathbf{0} \\
\mathbf{h}_{p}^{[1]}(1)^{T}
\end{array}\right]}_{\text {rank=1}} \mathbf{u}_{p}^{[2]}+\left[\begin{array}{c}
z_{p}^{[1]}(1) \\
z_{p}^{[1]}(2) \\
z_{p}^{[1]}(3)
\end{array}\right],
$$

where $\mathbf{0}$ is a $1 \times 2$ vector with all zeros. The desired signal space occupies 2 dimensions, while the interference is aligned in a one dimensional vector. Note that the interference due to transmission to user 2 can be measured over symbol extension 3 with the aim to subtract it at symbol extension 1 . Thus, the signal after zero forcing interference cancellation is

$$
\left[\begin{array}{c}
\widehat{y}_{p}^{[1]}(1) \\
\widetilde{y}_{p}^{[1]}(2)
\end{array}\right]=\left[\begin{array}{c}
\mathbf{h}_{p}^{[1]}(1)^{T} \\
\mathbf{h}_{p}^{[1]}(2)^{T}
\end{array}\right] \mathbf{u}_{p}^{[1]}+\left[\begin{array}{c}
z_{p}^{[1]}(1)-z_{p}^{[1]}(3) \\
z_{p}^{[1]}(2) .
\end{array}\right] .
$$

The same procedure can be followed to decode the symbols $\mathbf{u}_{p}^{[2]}$ transmitted to user 2. Therefore, each user achieves 2 DoF by solving a simple linear system during 3 symbol extensions. Consequently, $\frac{2}{3}$ DoF per user are attainable by employing BIA in the proposed toy example. However, beyond the DoF metric, the use of BIA involves a noise increment in the symbol extensions where simultaneous transmission is employed due to the interference subtraction. 
For the general case, assuming uniform power allocation to each symbol, the achievable sum rate of BIA in a OFDM system with $N_{\text {ofdm }}$ subcarriers is given by

$$
R_{\text {sum }}^{\text {BIA }}=\sum_{p=1}^{N_{\text {ofdm }}} \sum_{k=1}^{K} \log \operatorname{det}\left(\frac{1}{N_{t}+K-1} \mathbb{E}\left[\mathbf{I}+\tilde{\boldsymbol{P}} \overline{\mathbf{H}}_{p}^{[k]} \overline{\mathbf{H}}_{p}^{[k] H}\right]\right),
$$

where $\tilde{P}=\frac{\left(K+N_{t}-1\right)}{N_{t}^{2} K} P$ is the power assigned to each stream and

$$
\overline{\mathbf{H}}_{p}^{[k]}=\left[\begin{array}{llll}
\frac{\mathbf{h}_{p}^{[k]}(1)}{\sqrt{K}} & \ldots & \frac{\mathbf{h}_{p}^{[k]}\left(N_{t}-1\right)}{\sqrt{K}} & \mathbf{h}_{p}^{[k]}\left(N_{t}\right)
\end{array}\right]^{T} \in \mathbb{C}^{N_{t} \times N_{t}}
$$

is a full rank matrix.

\section{B. Linear Zero Forcing Beamforming}

With the aim to compare BIA with a transmission scheme based on CSIT, LZFB is taking into consideration. Assuming that each user maintains its channel mode fixed, if the channel $\mathbf{H}_{p}=\left[\mathbf{h}_{p}^{[1]}, \mathbf{h}_{p}^{[2]}, \ldots, \mathbf{h}_{p}^{[K]}\right] \in \mathbb{C}^{K \times N_{t}}$ is known at the transmitter, LZFB generates a precoding matrix given by

$$
\mathbf{W}_{p}=\mathbf{H}_{p}^{H}\left(\mathbf{H}_{p}^{H} \mathbf{H}_{p}\right)^{-1},
$$

where $\mathbf{W}_{p}=\left[\mathbf{w}_{p}^{[1]}, \mathbf{w}_{p}^{[2]}, \ldots, \mathbf{w}_{p}^{[K]}\right] \in \mathbb{C}^{N_{t} \times K}$ contains the precoding vectors $\mathbf{w}_{p}^{[k]} \in \mathbb{C}^{N_{t} \times 1}$ of the user $k$. By precoding the desired symbols $\mathbf{x}_{p}=\mathbf{w}_{p}^{[k]} \mathbf{u}_{p}^{[k]}$, the interference is entirely removed. In contrast with BIA, LZFB is only feasible if $K \leq N_{t}$, assuming that each user is equipped with only one antenna ${ }^{1}$. Since $N_{t}$ symbols can be transmitted during each time slot, LZFB achieves $N_{t}$ sum DoF.

The sum rate achieved by LZFB in a OFDM system with $N_{\text {ofdm }}$ subcarriers is given by

$$
R_{\text {sum }}^{L Z F B}=\sum_{p=1}^{N_{\text {ofdm }}} \sum_{k=1}^{K} \log \left(1+P \mathbf{w}_{p}^{[k]}{ }^{H} \mathbf{h}_{p}^{[k]}{ }^{H} \mathbf{h}_{p}^{[k]} \mathbf{w}_{p}^{[k]}\right),
$$

where $P$ is the power assigned to each stream assuming uniform power allocation. Note that channel estimation errors or phase synchronization drifts are not considered in the equation (8).

\section{Measurement Set-up}

A MISO BC is considered in the proposed testbed. The transmitter is equipped with $t=2$ antennas that serve 2 users equipped with a reconfigurable antenna each as is shown in Figure 3. The transmitter and the receivers are located in a lecture room at the University of Santander. The receivers are separated around six meters away from the transmitter with direct line of sight. Since the measurements are done in an isolated room, the coherence time is large enough to consider that the channel remains constant during each iteration. With the aim to simulate the behavior of reconfigurable antennas, two independent antennas from each node are taken into account. Therefore, two streams, which correspond to mode 1 and 2 , respectively, are received by each user.

\footnotetext{
${ }^{1}$ Note that a reconfigurable antenna employs only one RF chain, therefore, only one antenna can be employed when LZFB is put into practice.
}

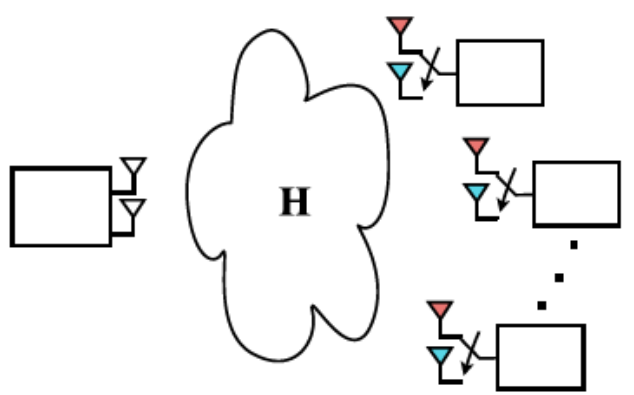

Fig. 3. Measurement configuration.

TABLE I

SYSTEM PARAMETERS

\begin{tabular}{|l|c|}
\hline Parameter & Value \\
\hline Signal bandwidth & $5 \mathrm{MHz}$ \\
\hline RF carrier & $5600 \mathrm{MHz}$ \\
\hline Number of subcarriers & 512 \\
\hline Subcarrier spacing & $15 \mathrm{KHz}$ \\
\hline Data subcarriers & 316 \\
\hline Null/Guard subcarriers & 32 \\
\hline Cyclic prefix & $1 / 8$ of the OFDM symbol length \\
\hline Sampling frequency & $40 \mathrm{Msamples} / \mathrm{sec}$ \\
\hline Sampling rate & $8 \mathrm{samples} / \mathrm{symbol}$ \\
\hline
\end{tabular}

The transmit and receive sides can be modeled as a combination of hardware and software parts. In [6], [8] these elements are described in detail. In contrast with other BIA implementations, the system parameters correspond to a LTE configuration for a $5 \mathrm{MHz}$ bandwidth and 512 subcarriers. In order to obtain a sampling frequency of $7.68 \mathrm{MHz}$ typical of this LTE configuration, a resampling factor is applied. The parameters of the testbed are shown in Table 1. In the presented implementation, the source bits can be mapped to a BPSK, QPSK, 16 QAM or 32 QAM constellation with one symbol per subcarrier. In this work, all the subcarriers of the OFDM symbol employ the same constellation in each iteration. This parameter fixes the sum-throughput of the proposed BIA implementation. The pilots symbols are always mapped to QPSK. Besides, LTE channel coding is assumed, a $\frac{2}{3}$ turboencoder is employed in this work. Regarding the noise, the testbed is characterized by an average SNR of $16 \mathrm{~dB}$.

\section{MEASUREMENT METHODOLOGY}

Two different methodologies are employed in this work. Figure 4 shows the frame structures employed in full CSIT and blind transmission schemes.

\section{A. Techniques based on CSIT}

The frame structure employed for LZFB transmission is shown in Figure 4(a). However, the same scheme can be employed for other techniques based on CSIT such as IA or BD. The frame structure is composed by a training stage, where sequential transmission is mandatory. It contains a PN sequence for synchronization and a pilot sequence (PLT) for channel estimation. This sequence corresponds to a entire OFDM symbol where the $N_{\text {of } d m}$ subcarriers contain a known 


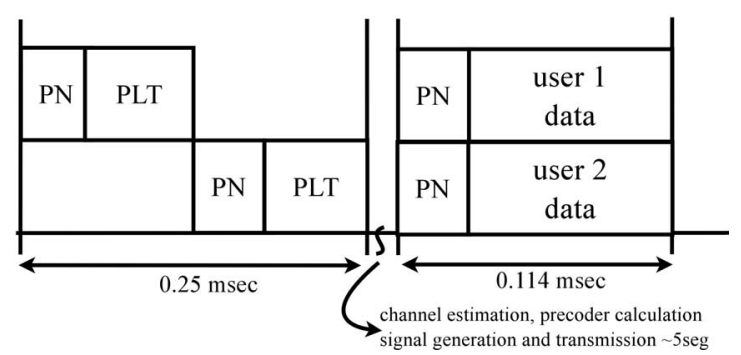

(a) Frame structure for LZFB (full CSIT)

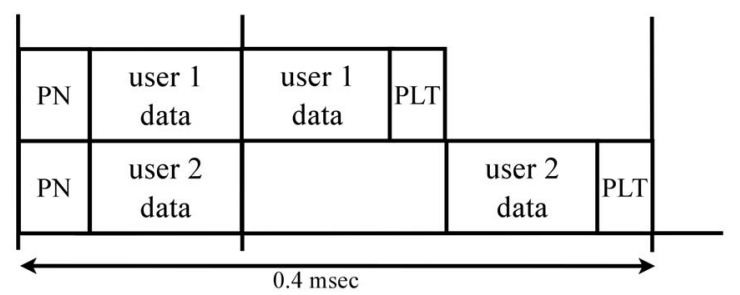

(b) Frame structure for BIA

Fig. 4. Frame structure for LZFB and BIA transmission schemes

QPSK symbol. Once the channel has been estimated, it is sent to the transmitters in order to calculate the corresponding precoding matrices and generate the data signals. This process involves about 5 seconds to be done. After that, 10 OFDM symbols are sent simultaneously to each user. Besides, since relative phases and time offsets have to be constant over the channel transmission, phase synchronization is required to implement full CSIT techniques.

\section{B. Blind Interference Alignment}

As other techniques that do not require CSIT knowledge, BIA implementation does not need training stage, backhaul links or phase synchronizations. As can be seen in Figure 4(b), the OFDM symbols are transmitted simultaneously during the first stage, which corresponds to first time slot (Block 1) of the supersymbol of Figure 2. After that, symbols to users 1 and 2 are transmitted in an orthogonal fashion according to the Block 2 of the supersymbol. Additionally, since sequential transmission is used during Block 2, a pilot sequence is also transmitted to get the CSIR required to solve the equation system of (4). Note that, although synchronization requirements are more relaxed for the BIA scheme, PN sequences are transmitted in order to detect the beginning of the frame.

\section{CSIT and CSIR overheads}

The most salient feature of BIA is the absence of CSIT. Therefore, the costs of implement full CSIT techniques should be taken into account when they are compared with BIA [9]. First, a frame with pilots is sent for channel estimation in an orthogonal fashion. Let $\theta_{c s i}$ denotes the ratio of pilots to the total number of subcarriers sent in the frame structure, a cost of $N_{t} \theta_{c s i}$ penalizes the sum rate achieved by a full CSIT technique. Additionally, the channel estimation has to be sent to the set of transmitters. Hence, an amount of the network resources are sacrificed to satisfy this point. The fraction of network resources allocated for feedback transmission to the total is denoted as $\theta_{f b}$. In both cases, full CSIT and BIA, once the data signal is received, pilots are typically required for coherent detection, these costs are denoted as $\theta_{c d}$. In this work, 10 data frames are transmitted after the training stage, and pilots occupy a whole OFDM symbol, therefore, the cost associated to CSI is $\theta_{c s i}=10 \%$. It is assumed that feedback overhead consumes $\theta_{f b}=2.5 \%$ of the network resources, and same cost for coherent transmission is considered.

\section{Results}

\section{A. Theoretical and achieved performance}

The theoretical achievable sum-rates of LZFB and BIA are shown in Figure 5. At first sight LZFB exceeds the performance of BIA. This result should not surprise us, since LZFB achieves 2 sum DoF while the performance of BIA is only $\frac{4}{3}$ sum DoF. For the average $\mathrm{SNR}=16 \mathrm{~dB}$ of our testbed, BIA achieves $4 \mathrm{bits} / \mathrm{sec} / \mathrm{Hz}$. Due to the LTE channel coding, the performance of our testbed is close to the capacity. Therefore, each user will obtain a bit error rate (BER) close to zero for a spectral efficiency of $2 \mathrm{bits} / \mathrm{sec} / \mathrm{Hz}$, i.e. by employing a QPSK constellation to transmit each stream. On the other hand, LZFB attains $5.5 \mathrm{bits} / \mathrm{sec} / \mathrm{Hz}$ for the same SNR. In consequence, from a theoretical point of view, it is expected that LZFB overcomes the performance of BIA.

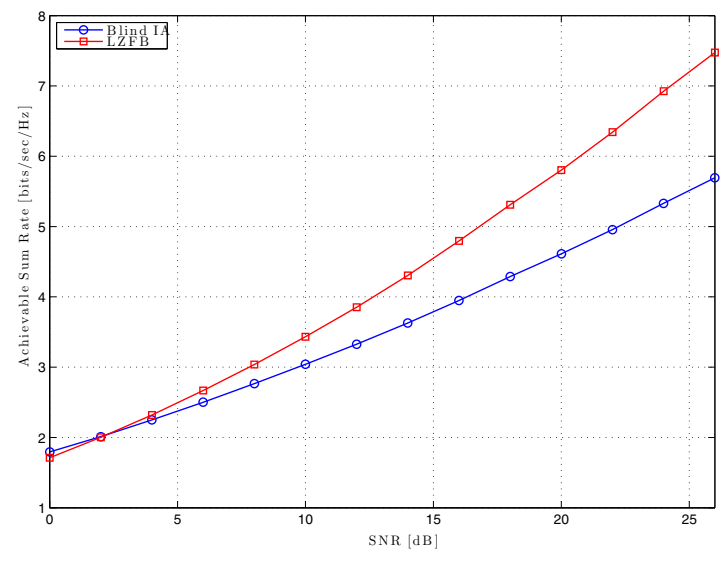

Fig. 5. Theoretical sum rate of LZFB and BIA in low SNR

The BER for LZFB and BIA transmission schemes is depicted in Figure 6. Note that the horizontal axis represents the sum-throughput taking into account the costs of CSIT and the structure of the frames shown in Figure 4. Each point corresponds to the constellation employed to map the transmitted symbols (BPSK, QPSK, 16-QAM, and 32-QAM), which determines the sum-throughput. It can be seen that LZFB achieves a lower BER for a sum-throughput below 12 Mbps. However, BIA obtains a better performance when more dense constellations are applied (e.g. when the symbols are mapped in a QPSK constellation, BIA achieves a BER of 
$3 \cdot 10^{-4}$ for $13.3 \mathrm{Mbps}$ while LZFB obtains a BER close to $1 \cdot 10^{-1}$ for $\left.15 \mathrm{Mbps}\right)$.

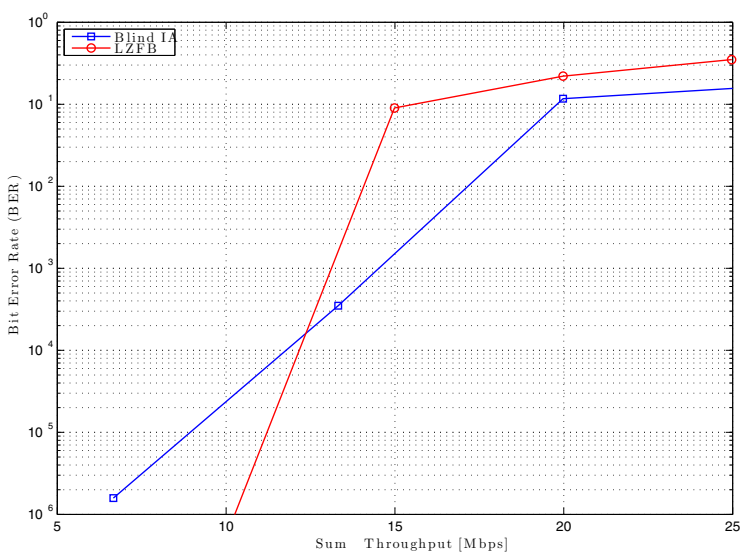

Fig. 6. BER vs sum throughput for LZFB and BIA shemes

\section{B. Discussion of the results}

Checking the results shown in Figure 6, the performance of BIA and LZFB can be confusing. According to the theoretical sum-rate shown in Figure 5, it was expected that LZFB overcomes the performance of BIA, however this only happens when a low density constellation such a BPSK is employed. This effect can be easily explain because of full CSIT schemes such as LZFB are heavily handicapped by channel estimation errors, synchronization drifts, and changes in the estimated channel, which do not depend directly on the SNR of the system. On the other hand, BIA is mainly limited by SNR. Therefore, it is expected that BIA achieves better performance than LZFB at higher SNR values in many practical environments, where the CSIT requirements are usually challenging to satisfy.

\section{CONCLUSIONS}

An experimental validation of BIA at low SNR regime is presented in this paper. It was shown that BIA achieves a reasonable performance assuming system parameters from mobile standards such as LTE. A comparison with LZFB shows that BIA exceeds the performance of a full CSIT technique in a wide range of rates. Furthermore, it is expected that even better results will be obtained at higher SNR regimes. Therefore, taking into account the requirements and network resources necessary to implement full CSIT techniques, BIA is an attractive transmission scheme when CSIT is not available or synchronization requirements cannot be fulfilled.

\section{REFERENCES}

[1] Tiangao Gou, Chenwei Wang, and S.A. Jafar, "Aiming perfectly in the dark-blind interference alignment through staggered antenna switching," IEEE Transactions on Signal Processing, vol. 59, no. 6, pp. 2734-2744, June 2011.
[2] R. Qian, M. Sellathurai, and D. Wilcox, "On the design of blind interference alignment using espar antenna," in Communications and Networking in China (CHINACOM), 2012 7th International ICST Conference on, Aug 2012, pp. 866-870.

[3] Tiangao Gou, S.A. Jafar, and C. Wang, "On the degrees of freedom of finite state compound wireless networks," Information Theory, IEEE Transactions on, vol. 57, no. 6, pp. 3286-3308, June 2011.

[4] O. El Ayach, S.W. Peters, and R.W. Heath, "The feasibility of interference alignment over measured mimo-ofdm channels," IEEE Transactions on Vehicular Technology, vol. 59, no. 9, pp. 4309-4321, Nov. 2010.

[5] O. González, D. Ramírez, I. Santamaría, J.A. García-Naya, and L. Castedo, "Experimental validation of interference alignment techniques using a multiuser mimo testbed," in Smart Antennas (WSA), 2011 International ITG Workshop on, Feb. 2011.

[6] M. Morales Céspedes, J. Gutiérrez Terán, and A. García Armada, "Achievable throughput with block diagonalization on ofdm indoor demonstrator," in 2013 European Signal Processing Conference (EUSIPCO 2013), September. 2013.

[7] K. Miller, A. Sanne, K. Srinivasan, and S. Vishwanath, "Enabling realtime interference alignment: Promises and challenges," MobiHoc 2012.

[8] L. Vielva, J. Via, J. Gutiérrez, O. González, J. Ibánez, and I. Santamaría, "Building a web platform for learning advanced digital communications using a mimo testbed," in Acoustics Speech and Signal Processing (ICASSP), 2010 IEEE International Conference on, March. 2010, pp. 2942-2945.

[9] Chenwei Wang, H.C Papadopoulos, S.A. Ramprashad, and G. Caire, "Improved blind interference alignment in a cellular environment using power allocation and cell-based clusters," in 2011 IEEE International Conference on Communications (ICC), June 2011, pp. 1-6. 